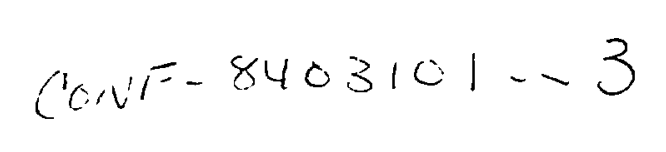

By acceptance of this article, the publisher or recipient dcknowledgas the U.S. Government's right to COHF $-8403101--3$ retoin a nonexclusive, royalty-tree license in and to any copyright covering the article.

\title{
Initial Energy Density of Quark-Gluon Plasma in Relativistic Heavy-Ion Collisions*
}

\author{
Cheuk-Yin Wong \\ Oak Ridge National Laboratory \\ Oak Ridge, TN 37831 \\ WINTER WORKSHOP ON NUCLEAR DYNAMICS III \\ Copper Mountain, Colorado \\ March 5-9, 1984
}

\section{DISCLAIMER}

This report was prepared as an account of work sponsored by an agency of the United States Government. Neither the United States Government nor any agency thereof, nor any of their employees, makes any warranty, express or implied, or assumes any legal liability or responsibility for the accuracy, completeness, or usefulness of any information, apparatus, product, or process disclosed, or represents that its use would not infringe privately owned rights. Reference herein to any specific commercial product, process, or service by trade name, trademark, manufacturer, or otherwise does not necessarily constitute or imply its endorsement, recommendation, or favoring by the United States Government or any agency therrof. The views and opinions of authors expressed herein do not necessurily state or reflect those of the United States Government or any agency thereof.

\footnotetext{
* Research sponsored by the Division of High Energy and Nuclear Physics, U.S. Department of Energy, under Contract W-7405-eng-26 with the Union Carbide Corporation.
} 
Initiai Energy Density of Quark-Gluon Plasma in Relativistic Heavy-Ion Collisions*

\author{
Cheuk-Yin Wong \\ 0ak Ridge National Laboratory, Oak Ridge, TN 37831
}

Recently, there is considerable interest in the central rapidity region of highly relativistic heavy-ion collisions. $1-5$ Such an interest stems from the possibility of creating hadron matter of high energy density which may exceed the critical energy density for a phase transition between ordinary confined matter and the unconfined quark-gluon plasma. ${ }^{6}$ The experimental searches and identification of the quark-gluon plasma may provide a new insight into the question of quark confinement. Furthermore, the creation of a domain of highenergy density may allow one to study matter under unusual conditions such as those which exist in the history of the early universe.

The estimate of the initial energy density is quite uncertain. The initial energy density is nonethe? ess an important physical quantity. It is one of the factors which determines whether the produced matter can undergo phase transition or not. In Ref. 1 it was assumed that in a central collision a nucleon in one nucleus only makes a single collision with nucleons in the other nucleus. The number of collisions a nucleon suffers in traversing the other nucleus has not been properly treated. The energy density has also been estimated previously by using the color neutralization model of Brodsky et a?. ${ }^{7}$ However, the color neutralization model gives a central rapidity muTtipTicity in heavy-ion collision too low by a factor of two. ${ }^{4}$ For this reason, we wish to obtain a better estimate of the energy density (in the central rapidity region).

As is well known, a simple Glauber-type multiple collision modei can reproduce the total multiplicity and multiplicity plateau near the central rapidity region to within $30 \% .8$ The simple multiple collision model has an approximate validity as a gross description of the reaction process. We shall adopt a semiempirical approach. Using the multiple collision model and the thickness function of Glauber, ${ }^{9}$ we obtain analytical functional form for all the quantities in question. A single parameter, $r_{r m s}$, is adjusted to fit the experimental central rapidity multiplicity data. The semi-empirical results provide a useful tool to extrapolate to the unknown central rapidity region of heavy-ion collisions.

In the multiple collision model, a nucleon in one nucleus makes many inelastic collisions with nucleons in the other nucleus, the probability of collision being given by the thickness function and the total nucleon-nucleon inelastic cross section. A nucleon may charige its identity during its passagae through the other nucleus, but its baryon number remains unchanged. Each nucleon-nucleon or baryon-baryon collision degrades the energies and momenta of the colliding baryons and produces particles outside the nucleus 10 in accordance with the experimental nucleon-nucleon particle production data. Thus, the initial mu?tiplicity distribution shortly after the two nuclei interpenetrate each other comes from nucleon-nucleon collisions in an additive manner. For a given configuration, the degree of this additivity is related to the number of nucleon-nucleon collisions. $n(b)$ when the two nuclei pass through each other. 
We discuss first the crudest approximation in which one takes the multiplicity distribution to be the same for each nucleon-nucleon or baryon-baryon collision. Then, the initial multiplicity distribution for the collision of nuclef $A$ and $B$ at an impact parameter $b$ is related to the multiplicity distribution for nucleon-nucleon collision $d N / d y$ for the same nucleon-nucleon center-ofmass energy by

$$
\frac{d N^{A B}}{d y}(b)=\frac{d N}{d y} n(b) \text {. }
$$

When we ayerage over the impact parameters, the average multiplicity distribution is given by

$$
\left\langle\frac{d N^{A B}}{d y}\right\rangle=\frac{d N}{d y} \cdot \frac{A B \sigma_{\text {in }}}{\sigma_{\text {in }}^{A B}},
$$

where $\sigma_{i n}$ is the nucleon-nucleon inelastic cross section and $\sigma_{i n}^{A B}$ is the nucleusnucleus inelastic cross section.

With a Gaussian form of the thickness function, we obtain the following functional form for the multiplicity distribution

$$
\frac{d N^{A B}}{d y}(b)=\frac{d N}{d y} \frac{A B f e}{\left[1-(1-f e)^{A B}\right]},
$$

where

$$
\begin{aligned}
& f=\sigma_{i n} / 2 \pi \beta^{2}, \\
& e=\exp \left[-b^{2} / 2 \beta^{2}\right],
\end{aligned}
$$

and $B^{2}=r_{r m s}^{2}\left(A^{2 / 3}+B^{2 / 3}\right) / 3+B_{p}^{2}$.

Here, $r_{r m s}$ is the root-mean-square radius parameter and $\beta_{p}=0.68 \mathrm{fm}$ is the standard deyiation of the nucleon-nucleon thickness function. The functional form of the ratio of the average multiplicity distributions is then

$$
R\left(\frac{A B}{p p}, y\right)=\frac{\left\langle\frac{d N^{A B}}{d y}\right\rangle}{\frac{d N}{d y}}=\frac{A B f}{\sum_{i=1}^{A B}\left[1-(1-f)^{i}\right] / i} .
$$

If we mindlessly apply Eqs. (3) and (4) by using the root-mean-squared radius parameter as determined by electron scattering, the theoretical results consistently exceed the experimental values. In order to correct for this 
systematic discrepancy, wo shall adopt a semi-empirical approach. This consists of assuming the functiona form of Eqs. (3) and (4) with its only parameter $r_{r m s}$ so chosen as to fit the available central rapidity multiplicity data. We fourd that $r_{\text {rms }}=1.15 \mathrm{fm}$ gives good fits to the experimental central multiplicity data $11-13$ of $p \alpha$, dd, $\alpha \alpha, S i+A g$, and $C a+C$ (Table I). The results of Eqs. (3) and (4) can be used to discuss the collisions of other nuclei.

Using the relationship between $d N^{A B} / d y$ and the energy density $\varepsilon$ as given by Ref. 1 , we can calculate the energy density in the central rapidity region. The energy density $\varepsilon(b)$ in the collision of nuclei $A$ and $B$ with an impact parameter $b$ is given by

$$
\frac{\varepsilon(b)}{\varepsilon_{0}}=\frac{A B T(b) \sigma_{\text {in }}}{\left\{1-\left[1-T(b) \sigma_{\text {in }}\right]^{A B}\right\} \mathscr{A ( b )}}
$$

where $T(b)$ is the normalized thickness function and can be approximated by

$$
T(b)=\exp \left[-b^{2} / 2 \beta^{2}\right] / 2 \pi \beta^{2},
$$

\begin{tabular}{|c|c|c|c|}
\hline \multicolumn{2}{|c|}{ Huclef } & \multicolumn{2}{|c|}{$\left(d A^{A B} / d y\right) /(d N / G y)$} \\
\hline A & B & $\exp$ & Theory \\
\hline $\mathbf{p}$ & $p$ & 1 & 1 \\
\hline$g$ & a & $1.18 \pm 0.07$ & 1.19 \\
\hline d & d & $1.4 \pm 0.10$ & 1.42 \\
\hline$\alpha$ & $\alpha$ & $1.74 \pm 0.09$ & 1.71 \\
\hline si & Ag & $\sim 95$ & 96.0 \\
\hline Ca & c & $\sim 25$ & 27.1 \\
\hline
\end{tabular}

Table 1

$\mathscr{A}(\mathrm{b})$ is the transverse overlap area of the two nuclei, and $\varepsilon_{0}$ is a bombardingenergy-dependent unit of energy density. At the time of $1 \mathrm{fm} / \mathrm{c}$ after the nuclei collide with each other, particles are produced. The energy density unit $\varepsilon_{0}$ at that time is $0.84 \mathrm{GeV} / \mathrm{fm}^{3}$ for $\sqrt{\mathrm{s}} / \mathrm{A}=11.8 \mathrm{GeV}, 1.14 \mathrm{GeV} / \mathrm{fm}^{3}$ for $\sqrt{\mathrm{s}} / \mathrm{A}=31.4 \mathrm{GeV}$, and $1.80 \mathrm{GeV} / \mathrm{fm}^{3}$ for $\sqrt{\mathrm{s}} / \mathrm{A}=270 \mathrm{GeV}$. We calculate the initial energy density for head-on collisions of two equal nuclei. In Fig. 1 we show this quantity as a function of $A$. The numerical result can be parameterized as

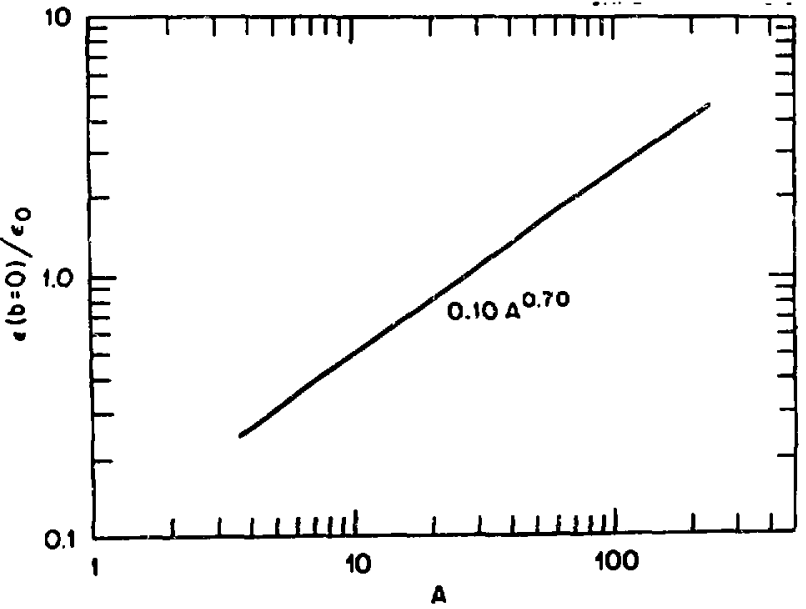

Figure 1

$$
\frac{\varepsilon(b=0)}{\varepsilon_{0}}=0.10 \mathrm{~A}^{0.709}
$$

which goes approximately as $A^{2 / 3}$, as expected from $\mathrm{Eq}$. (5). We also calculate the energy density in units of $\varepsilon_{0}$ as a function of the impact parameter for the collision of $238 \mathrm{U}$ on $238 \mathrm{U}$. The results are shown in Fig. 2. The energy density is about 5 units of $\varepsilon_{0}$. It oscillates as a function of the impact parameter with an amplitude of about $0.5 \varepsilon_{0}$. Our energy density estimates are greater than those of the previous estimates ${ }^{1,4}$ as the detailed treatments are different. At an incident energy of $J s / A=30 \mathrm{GeV}$ per nucieon, 
this corresponds to an energy density of about $5 \mathrm{GeV} / \mathrm{fm}^{3}$ which exceeds the energy density of $2 \mathrm{GeV} / \mathrm{fm}^{3}$ estimated to be the critical energy density for a phase transition. ${ }^{4}$

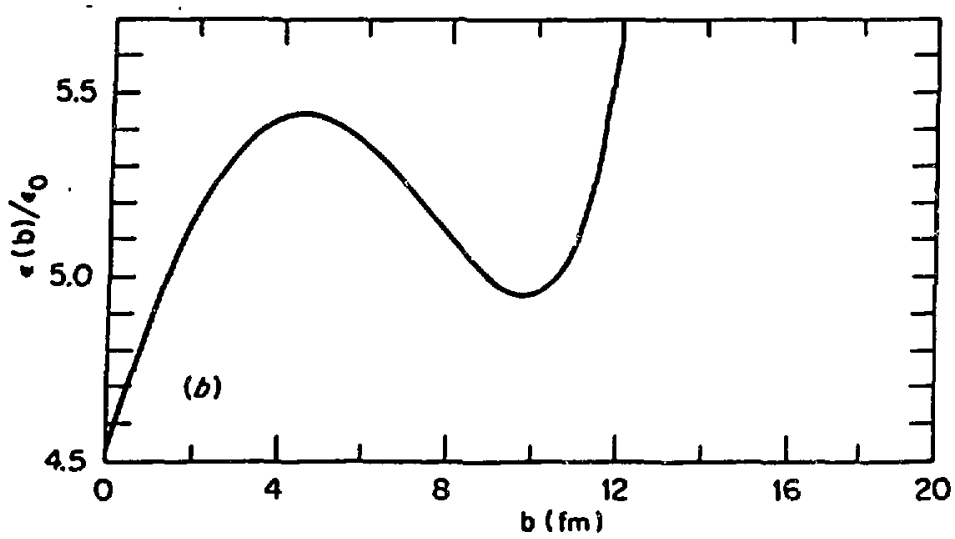

Figure 2

\section{REFERENCES}

* Research sponsored by the Division of High Energy and Nuciear Physics, I.S. Department of Energy, under contract W-7405-eng-26 with the Union Carbide Corporation.

1. J. D. Bjorken, Phys. Rev. D 27, 140 (1983).

2. K. Kajantie and L. McLerran, Phys. Lett. 119B, 203 (1982).

3. T. D. Lee, Coiumbia University Report No. CU-TP-226, 1981 .

4. M. Gyulassy, Lawrence Berkeley Report No. LBL-15175, 1982.

5. J. Rafelski and M. Danos, N.B.S. Report No. MBSIR 83-2725, 1983.

6. For example, L. McLerran and B. Svetitsky, Phys. Lett. 96B, 195 (1981); Phys. Rev. D 24, 450 (1981); L. Montvay and H. Pietarinen, Phys. Lett. 115B, 151 (1982); J. Kogut et al. 2 Phys. Rev. Lett. 48,1140 (1982).

7. S. J. Brodsky, J. F. Gunion, and J. H. Kuhn, Phys. Rev. Lett. 39,1120 (1977).

8. J. E. Elias, W. Busza, C. Halliwell, D. Luckey, L. Votta, and C. Young, Phys. Rev. Lett. 41, 285 (1978); J. E. Elias, W. Busza, C. Halliwell, D. Luckey, L. Votta, and C. Young, Phys. Rev. D 22, 13 (1980).

9. R. J. Giauber, Lectures in Theoretical Physics, ed. by W. E. Brittin and L. G. Dunham, Vol. 1, p. 315, Interscience, New York (1959).

10. W. Busza, Proceedings of 4th High Energy Heayy Ion Summer Study, June 1978, LBL-7766, p. 253 (unpublished).

11. W. Bell et al.: Proceedings of 5th Higli Energy Heavy Ion Study, Berkeley, May 1981, p. 540 .

12. M. Jacobs, Proceedings of 5th High Energy Heavy Ion Study, May 1981, LBL-12652, p. 581 (unpublished).

13. T. H. Burnett et al... Phys. Rev. Lett. 50, 2062 (1983). 